\title{
Compromiso de médula ósea en pacientes con linfoma B difuso de célula grande
}

\author{
Bone marrow involvement in patients with diffuse large B-cell lymphoma
} \author{
Luis Eduardo Pino² \\ ' Oncólogos Asociados de Imbanaco (Cali, Colombia). \\ 2 Servicio de Hematología y Oncología, Hospital Militar Central, Universidad Militar Nueva Granada (Bogotá, Colombia). \\ ${ }^{3}$ Facultad de Medicina, Departamento de Epidemiología, Universidad El Bosque (Bogotá, Colombia). \\ ${ }^{4}$ Servicio de Hematología y Trasplante de Médula Ósea, Hospital Pablo Tobón Uribe (Medellín, Colombia). \\ 5 Grupo Hematología y Trasplante de Médula Ósea, Fundación Santa Fe de Bogotá (Bogotá, Colombia).
}

Milton Alberto Lombana1-3, Juan Felipe Combariza4, Ana Milena Torres³, Javier Segovia², Andrés Acevedo ${ }^{5}$,

\section{Resumen}

Introducción: El compromiso de médula ósea concordante en pacientes con linfoma B difuso de célula grande se asocia a un peor pronóstico, sin embargo, el uso de rituximab parece disminuir este riesgo. Se desconoce si el compromiso de médula ósea es un factor predictor de respuesta o un factor pronóstico directo para supervivencia en población latinoamericana en la era del rituximab.

Diseño y métodos: Estudio multicéntrico de cohorte dinámica pronóstica de pacientes colombianos con diagnóstico de linfoma B difuso de célula grande. El objetivo principal fue determinar el papel del compromiso tumoral de médula ósea como factor predictivo en términos de respuesta al tratamiento y como factor pronóstico independiente para supervivencia libre de eventos y supervivencia global a dos años. El análisis de supervivencia se realizó por el método no paramétrico de KaplanMeier. La comparación de probabilidad de supervivencia entre los grupos se hizo con el test Long-Rank.

Resultados: Se incluyeron 163 pacientes con linfoma B difuso de célula grande. La prevalencia del compromiso de médula ósea fue del $11 \%$. La respuesta completa fue del $77 \%$ versus el $38 \%$ en pacientes sin y con compromiso de médula ósea respectivamente ( $p<0,0001$ Fisher). El análisis multivariado demostró que el compromiso de médula ósea se asoció significativamente con menor probabilidad de obtener respuesta al tratamiento. La respuesta al tratamiento y el puntaje IPI bajo disminuyeron el riesgo de recaída. El análisis multivariado por regresión de Cox mostró que solo la respuesta al tratamiento y el IPI alto afectaron de manera significativa la supervivencia general. En pacientes con IPI de riesgo bajo y respuesta objetiva, la supervivencia a dos años fue del $99 \%$ y del $17 \%$ en pacientes con IPI alto y sin respuesta. El análisis por Kaplan-Meier evidenció que no fue el compromiso por médula ósea sino la respuesta al tratamiento lo que influenció la supervivencia. Conclusiones: Nuestro estudio confirma el valor predictivo significativo del compromiso de médula ósea para la pobre respuesta al tratamiento. La supervivencia global solo fue impactada por el puntaje IPI y la respuesta al tratamiento, evidenciando que el compromiso de médula ósea parece ser un factor predictivo de respuesta y no pronóstico independiente para supervivencia global.

Palabras clave: linfoma, médula ósea, mieloptisis, pronóstico.

\begin{abstract}
Introduction: bone marrow involvement in patients with diffuse large B-cell lymphoma is associated with a worse prognosis; however, the use of rituximab appears to improve it. It is unknown whether myelophthisis is a predictor of response or a prognostic factor for survival in Latin America during the rituximab era.

Design and Methods: The aim of this multicenter dynamic prognostic cohort study was to determine the role of lymphoma bone marrow involvement to predict response to treatment and its usefulness as an independent prognostic factor for event free survival and overall survival. The survival analysis was performed by the nonparametric Kaplan-Meier method. Comparison of probability of survival between groups was made using the log-rank test.

Results: 163 patients with diffuse large cell B lymphoma were included. The prevalence of myelophthisis was $11 \%$. Complete response was $77 \%$ versus $38 \%$ in patients with and without bone marrow involvement respectively $(\mathrm{P}<0.0001$ Fisher). Multivariate analysis showed that bone marrow involvement was significantly associated with less likelihood of treatment response. Treatment response and low IPI score reduced the risk of relapse. Multivariate analysis by Cox regression showed that only treatment response and high IPI significantly affect overall survival. In patients with low-risk IPI and objective response and in those with high IPI and no response the two-year survival was 99\% and 17\% respectively. The Kaplan-Meier analysis showed that treatment response was the only variable that influenced survival.
\end{abstract}


Conclusions: Our study confirms the significant predictive value of bone marrow involvement for poor response treatment. Overall survival was only modified by the IPI score and treatment response. Bone marrow involvement seems to be a predictor of response and no an independent prognostic factor for overall survival.

Keywords: lymphoma, bone marrow, myelophthisis, prognosis.

\section{Introducción}

En Colombia, los linfomas no Hodgkin constituyen la octava causa de incidencia por cáncer, con 2.309 casos nuevos (5,8 por cada 100.000 habitantes) y una mortalidad de 986 casos (2,5 por cada 100.000 habitantes) para el 20081. El pronóstico de SG y supervivencia libre de recaída dependen de diferentes factores, de los cuales el puntaje IPI es el más relevante, estimando una probabilidad de supervivencia a tres años del $91 \%$ si el puntaje es de riesgo bajo y de un $59 \%$ en pacientes con riesgo alto ${ }^{2-4}$. Dentro de otros factores, el compromiso de médula ósea parece ser deletéreo para la supervivencia global (SG) y la supervivencia libre de recaída, especialmente si la histología del compromiso es concordante ${ }^{5,6}$. Sin embargo, esta asociación no es consistente en todas las poblaciones reportadas, principalmente cuando se usa rituximab ${ }^{6-11}$.

Gaudio y colaboradores compararon un grupo de pacientes en Italia con linfoma B difuso de célula grande (LBDCG) tratados con CHOP con y sin rituximab, y concluyeron que el uso de este disminuía el riesgo desfavorable del compromiso tumoral en médula ósea ${ }^{11}$. Un hallazgo similar fue reportado por Sh y colaboradores en población china ${ }^{10}$. Contrario a estos reportes, un estudio cooperativo con población canadiense y de Nueva Zelandia demostró que, en pacientes cuyo tratamiento incluía rituximab, el compromiso de médula ósea afecta de manera significativa la supervivencia general y la supervivencia libre de progresión ${ }^{5}$. Según nuestro conocimiento, no existen estudios que evalúen el valor pronóstico del compromiso de médula ósea en pacientes con linfoma B difuso de célula grande en población colombiana ni latinoamericana tratados con y sin rituximab.

\section{Diseño y métodos}

Se realizó un estudio multicéntrico de cohorte dinámica pronóstica retrospectivo de pacientes colombianos con diagnóstico de linfoma B difuso de célula grande, manejados en el Hospital Militar Central de la ciudad de Bogotá, el Hospital Pablo Tobón Uribe de Medellín, la Fundación Santa Fe de Bogotá y el grupo de
Oncólogos Asociados de Imbanaco en Cali. El objetivo principal fue determinar el papel del compromiso tumoral concordante en médula ósea como factor predictivo en términos de respuesta al tratamiento y como factor pronóstico independiente para supervivencia libre de eventos y SG a dos años. En estas instituciones, a todos los pacientes con diagnóstico de linfoma $B$ difuso de célula grande se les hace un estudio de médula ósea como protocolo institucional durante su estadificación inicial. Para propósitos de este análisis, solo se incluyeron pacientes con compromiso concordante de médula ósea, debido a que su implicación pronóstica comparada con el compromiso discordante ya está ampliamente confirmada.

La información se obtuvo de las historias clínicas de pacientes atendidos en el servicio de hematología y oncología de las cuatro instituciones. Los reportes de confirmación del compromiso de médula ósea concordante se obtuvieron de los reportes de patología registrados o consignados en las historias clínicas. La respuesta al tratamiento se definió de acuerdo con the International Working Group response criteria for malignant lymphomas 2007, según lo registrado en la historia clínica por el médico tratante, corroborado con los datos imaginológicos disponibles y evaluados por los investigadores de manera ciega a los desenlaces y las características de cada paciente, en caso de ser necesario. Se definió evento como recaída, progresión o muerte, calculándose el tiempo de supervivencia libre de evento desde el diagnóstico hasta la comprobación clínica, imaginológica o histológica de este. La fecha de supervivencia libre de recaída se calculó desde la fecha de la comprobación de la respuesta objetiva al tratamiento hasta la fecha de comprobación imaginológica y/o histológica de recaída del linfoma; y el tiempo de SG, desde el diagnóstico histopatológico hasta la fecha de comprobación de muerte registrada en la historia clínica o en el libro de defunciones del servicio. En caso de no presentar ningún evento, los datos fueron censurados al 23 de marzo del 2013.

Para propósitos del análisis multivariado por regresión logística y modelo de Cox, el puntaje IPI se 
dicotomizó como riesgo alto ( $\geq 3$ ) y bajo (0-2). El contraste de hipótesis y las pruebas de independencia se analizaron mediante chi cuadrado o prueba exacta de Fisher según se requiriese. La asociación entre respuesta al tratamiento y las variables analizadas se efectuó por riesgo relativo y odds ratio en el análisis multivariado. El análisis de supervivencia se efectuó por el método no paramétrico de Kaplan-Meier. La comparación de probabilidad de supervivencia entre los grupos se hizo con el test Long-Rank o Wilcoxon según se requiriese. La asociación entre las variables dependientes de tiempo y el modelo de análisis multivariado se llevó a cabo por medio de hazard ratio con el método de Cox. Se estableció un nivel de significancia del $5 \%$ con cálculo de intervalos de confianza del 95\%. Se usó el programa Stata 11.2 para el análisis estadístico. El estudio fue aprobado por el comité de investigaciones de la Universidad Militar Nueva Granada.

\section{Resultados}

Se incluyeron 164 pacientes con linfoma B difuso de célula grande, diagnosticados entre enero del 2000 y diciembre del 2012. La prevalencia del compromiso de médula ósea fue del $11 \%$. Esta prevalencia fue más alta, del $33 \%$, en pacientes con infección por $\mathrm{VIH}(p=0,056)$; sin embargo, el número de pacientes con VIH incluidos fue pequeño, solo 9. Del total, 126 (78\%) utilizaron rituximab y 36 (22\%) no lo recibieron debido a que fueron diagnosticados en fechas previas a la aprobación de su uso en Colombia. Las características demográficas de la población se describen en la tabla 1.

\section{Respuesta al tratamiento}

En la población total, considerando el tratamiento de primera línea, la respuesta completa fue del $73 \%$, respuesta parcial del $11 \%$ y no hubo respuesta en el $16 \%$ de los pacientes. La respuesta completa fue del $77 \%$ versus el $38 \%$ en pacientes sin y con compromiso de médula ósea, respectivamente. La no respuesta ocurrió en el $8 \%$ versus el $39 \%$ en pacientes sin y con médula comprometida, respectivamente ( $p<0,0001$ Fisher). En pacientes con médula ósea comprometida, el uso de rituximab mejoró la respuesta completa al $43 \%$ en aquellos con compromiso, comparado con un $25 \%$ en aquellos que no lo usaron ( $p=$ 0,03 Fisher). El análisis multivariado por regresión logística demostró que el compromiso de médula ósea incrementó de manera significativa e independiente el riesgo de no respuesta al tratamiento (tabla 2). El modelo multivariado tuvo un nivel de significancia con $p=0,0015$ y la prueba de bondad de ajustes fue de 0,536.

\section{Supervivencia libre de evento}

La supervivencia libre de evento (SLE) a dos años fue del 76\% (IC95\% 68-83\%) en la población total. El análisis univariado demostró que el compromiso de médula ósea, la enfermedad bultosa, los síntomas B, la infección por VIH, el IPI alto y la respuesta objetiva

Tabla 1. Características demográficas de la población en estudio

\begin{tabular}{|c|c|c|c|c|}
\hline $\begin{array}{l}\text { Característica } \\
\qquad(n=163)\end{array}$ & Total & $\begin{array}{c}\text { Sin compromiso } \\
\text { de médula ósea }(n=97)\end{array}$ & $\begin{array}{l}\text { Con compromiso } \\
\text { de médula ósea }(n=18)\end{array}$ & $\begin{array}{c}\mathbf{P} \\
\text { (Fisher) }\end{array}$ \\
\hline $\begin{array}{l}\text { Grupo de edad } \\
<64 \text { años } \\
64-79 \text { años } \\
>80 \text { años }\end{array}$ & $\begin{array}{c}113(69,75 \%) \\
41(25,31 \%) \\
8(4,94 \%)\end{array}$ & $\begin{array}{c}105(72,92 \%) \\
33(22,92 \%) \\
6(4,17 \%)\end{array}$ & $\begin{array}{l}8(44,44 \%) \\
8(44,44 \%) \\
2(11,11 \%)\end{array}$ & $p=0,022$ \\
\hline $\begin{array}{l}\text { Sexo } \\
\text { Femenino } \\
\text { Masculino }\end{array}$ & $\begin{array}{l}70(43,21 \%) \\
92(56,79 \%)\end{array}$ & $\begin{array}{l}63(43,75 \%) \\
81(56,25 \%)\end{array}$ & $\begin{array}{c}7(38,89 \%) \\
11(61,11 \%)\end{array}$ & $p=0,695$ \\
\hline $\begin{array}{l}\text { Infección por VIH } \\
\text { No } \\
\text { Sí }\end{array}$ & $\begin{array}{l}151(94,38 \%) \\
9(5,63 \%)\end{array}$ & $\begin{array}{c}137(95,80 \%) \\
6(4,20 \%)\end{array}$ & $\begin{array}{l}14(82,35 \%) \\
3(17,65 \%)\end{array}$ & $p=0,056$ \\
\hline $\begin{array}{l}\text { Síntomas B } \\
\text { No } \\
\text { Sí }\end{array}$ & $\begin{array}{l}78(48,15 \%) \\
84(51,85 \%)\end{array}$ & $\begin{array}{l}73(50,69 \%) \\
71(49,31 \%)\end{array}$ & $\begin{array}{c}5(27,78 \%) \\
13(72,22 \%)\end{array}$ & $p=0,082$ \\
\hline $\begin{array}{l}\text { Enfermedad bultosa (Bulky } \\
\text { No } \\
\text { Sí }\end{array}$ & $\begin{array}{l}107(66,05 \%) \\
55(33,95 \%)\end{array}$ & $\begin{array}{l}93(64,58 \%) \\
51(35,42 \%)\end{array}$ & $\begin{array}{l}14(77,78 \%) \\
4(22,22 \%)\end{array}$ & $p=0,305$ \\
\hline $\begin{array}{l}\text { IPI alto } \\
0-2 \\
>3\end{array}$ & $\begin{array}{l}98(60,87 \%) \\
63(39,13 \%)\end{array}$ & $\begin{array}{l}94(62,28 \%) \\
50(34,72 \%)\end{array}$ & $\begin{array}{c}4(23,53 \%) \\
13(76,47 \%)\end{array}$ & $p=0,001$ \\
\hline $\begin{array}{l}\text { Uso de rituximab } \\
\text { No } \\
\text { Sí }\end{array}$ & $\begin{array}{l}36(22,22 \%) \\
126(77,78 \%)\end{array}$ & $\begin{array}{c}32(22,22 \%) \\
112(77,78 \%)\end{array}$ & $\begin{array}{c}4(22,22 \%) \\
14(77,78 \%)\end{array}$ & $p=1,000$ \\
\hline $\begin{array}{l}\text { Uso de radioterapia } \\
\text { No } \\
\text { Sí }\end{array}$ & $\begin{array}{l}115(70,99 \%) \\
47(29,01 \%)\end{array}$ & $\begin{array}{l}101(70,14 \%) \\
43(29,86 \%)\end{array}$ & $\begin{array}{l}14(77,78 \%) \\
4(22,22 \%)\end{array}$ & $p=0,592$ \\
\hline
\end{tabular}


Tabla 2. Fallo (no respuesta) al tratamiento (análisis bivariado y multivariado)

\begin{tabular}{|c|c|c|c|c|c|c|}
\hline \multirow{2}{*}{ Variable } & \multicolumn{3}{|c|}{ Análisis bivariado } & \multicolumn{3}{|c|}{ Análisis multivariado (regresión logística) } \\
\hline & $\mathbf{R R}$ & IC95\% & Valor $p$ & OR & IC95\% & Valor $\mathrm{p}$ \\
\hline IPI alto & 2,09 & $0,93-4,68$ & 0,0659 & 1,33 & $0,46-3,81$ & 0,596 \\
\hline Compromiso de médula ósea & 4,92 & $2,36-10,23$ & $<0,00001$ & 8,53 & $2,41-30,2$ & 0,001 \\
\hline Edad $>65$ años & 0,74 & $0,29-1,92$ & 0,5436 & -- & -- & -- \\
\hline Sexo masculino & 0,68 & $0,30-1,52$ & 0,3493 & 0,47 & $0,11-1,97$ & 0,306 \\
\hline Infección por VIH & 3,97 & $1,68-9,36$ & 0,017 (Fisher) & 4,09 & $0,82-20,25$ & 0,084 \\
\hline Síntomas B & 1,00 & $0,45-2,24$ & 0,9817 & -- & -- & -- \\
\hline Estadio avanzado (III/IV) & 3,71 & $1,30-10,55$ & 0,0054 & -- & - & -- \\
\hline Enfermedad bultosa & 1,78 & $0,80-3,94$ & 0,1496 & 2,45 & $0,85-7,03$ & 0,096 \\
\hline Uso de rituximab & 0,70 & $0,29-1,69$ & 0,4427 & -- & - & -- \\
\hline Uso de radioterapia & 0,58 & $0,12-1,33$ & 0,1148 & -- & - & -- \\
\hline
\end{tabular}

Tabla 3. Supervivencia global (análisis bivariado y multivariado)

\begin{tabular}{|c|c|c|c|c|c|c|}
\hline \multirow{2}{*}{ Variable } & \multicolumn{3}{|c|}{$\begin{array}{l}\text { Análisis bivariado } \\
\text { por Kaplan-Meier }\end{array}$} & \multicolumn{3}{|c|}{$\begin{array}{l}\text { Análisis multivariado } \\
\text { (regresión de Cox) }\end{array}$} \\
\hline & HR & IC95\% & $\begin{array}{c}\text { Valor } p \\
\text { (Log-Rank) }\end{array}$ & HR & IC95\% & Valor $p$ \\
\hline IPI alto & 3,08 & $1,57-6,02$ & 0,0005 & 2,99 & $1,22-7,31$ & 0,016 \\
\hline Compromiso de médula ósea & 3,70 & $1,58-8,65$ & 0,0011 & 0,88 & $0,30-2,57$ & 0,819 \\
\hline Edad > 65 años & 1,00 & $0,48-2,06$ & 0,9986 & -- & -- & - \\
\hline Sexo masculino & 1,28 & $0,65-2,52$ & 0,4654 & - & -- & - \\
\hline Infección por VIH & 3,90 & $1,51-10,08$ & 0,0024 & 3,61 & $1,24-10,50$ & 0,018 \\
\hline Síntomas B & 2,01 & $1,01-4,01$ & 0,0415 & 1,11 & $0,45-2,69$ & 0,815 \\
\hline Enfermedad bultosa & 2,13 & $1,11-4,07$ & 0,0186 & 0,98 & $0,38-2,53$ & 0,970 \\
\hline Uso de rituximab & 0,64 & $0,32-1,28$ & 0,2083 & 0,95 & $0,44-2,04$ & 0,909 \\
\hline Uso de radioterapia & 1,26 & $0,64-2,48$ & 0,4930 & -- & -- & - \\
\hline Respuesta objetiva al tratamiento & 11,15 & $5,41-22,99$ & $<0,00001$ & 12,14 & $5,09-28,95$ & $<0,0001$ \\
\hline
\end{tabular}

$p$ (modelo) $<0,00001$ prueba de asunción de riesgos proporcionales Schoenfeld $=0,3976$

al tratamiento se asociaron con mayor probabilidad de recaída, siendo solo los tres últimos estadísticamente significativos en el modelo multivariado por regresión de Cox (tabla 3). La probabilidad de SLE a dos años fue del $82 \%$ en pacientes con IPI bajo que obtuvieron respuesta, y solo del $13 \%$ en pacientes con IPI alto y sin respuesta objetiva (figuras 1 a 3 ).

\section{Supervivencia global}

La SG a dos años para toda la población fue del 82\% (IC95\% 74-87\%). En el análisis univariado, el compromiso de médula ósea, la infección por VIH, el puntaje IPI alto, la no respuesta objetiva y la recaída/progresión se asociaron a mayor riesgo de muerte. El modelo multivariado de Cox demostró que solo la respuesta al tratamiento y el puntaje IPI afectaron de manera significativa la probabilidad de SG (tabla 4). En pacientes con IPI de riesgo bajo y respuesta objetiva, la supervivencia a dos años fue del $99 \%$ y solo del $17 \%$ en pacientes con IPI alto y sin respuesta (figura 4). Del mismo modo, el análisis por Kaplan-Meier evidenció que no fue el compromiso por médula ósea sino la respuesta al tratamiento lo que influenció la probabilidad de SG.

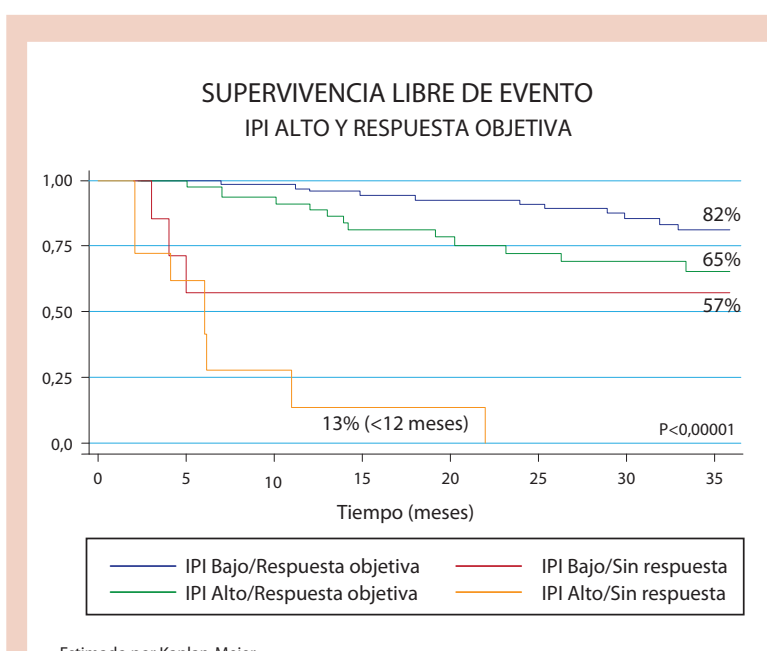

Estimado por Kaplan-Meier

Figura 1. Supervivencia libre de evento a dos años por compromiso de médula ósea y respuesta al tratamiento. 


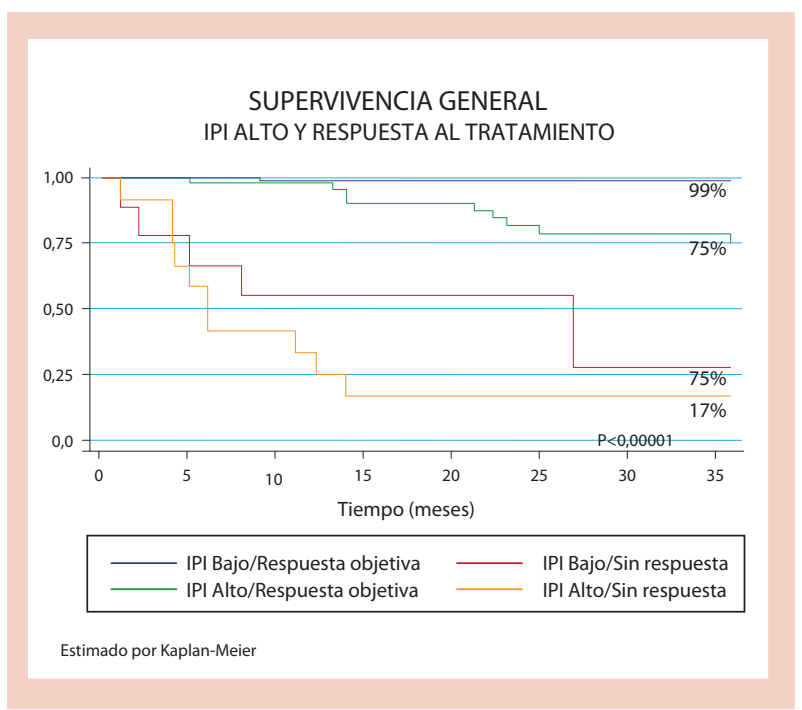

Figura 2. Supervivencia global a dos años por IPI y respuesta al tratamiento.

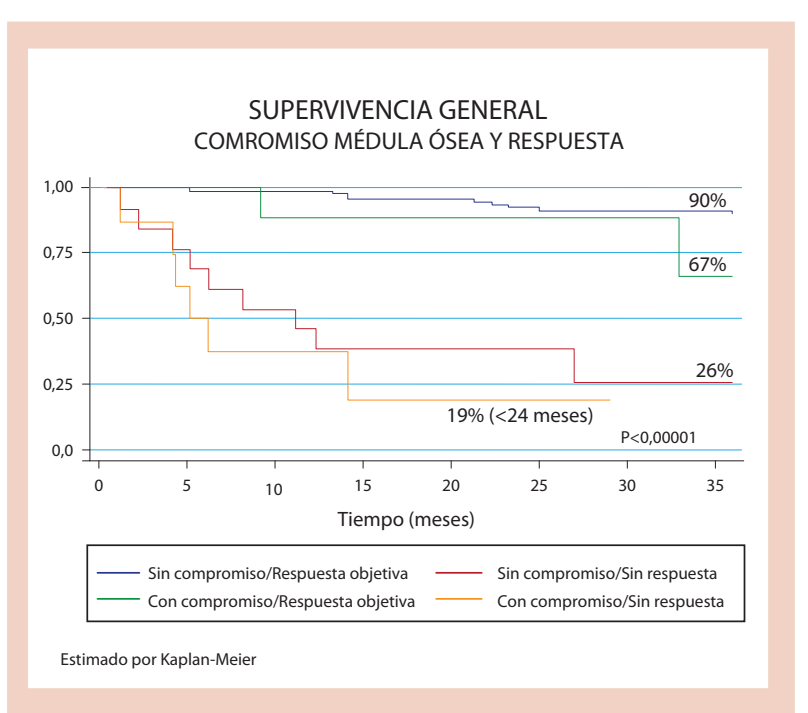

Figura 3. Supervivencia global por compromiso de médula ósea y respuesta al tratamiento.

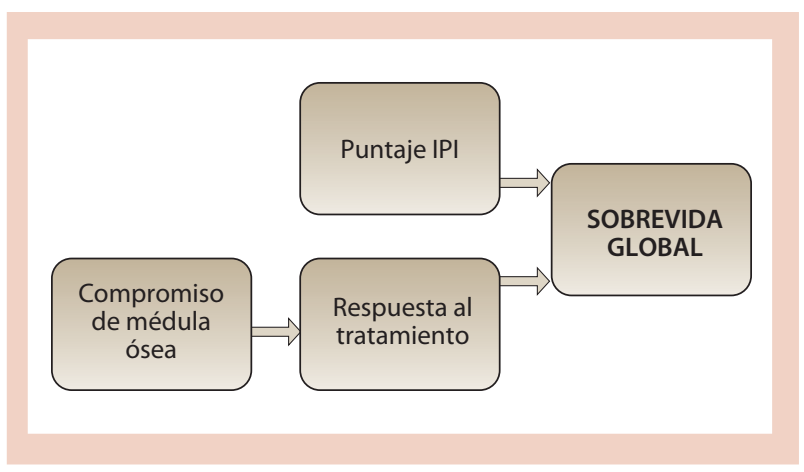

Figura 4. Relación hipotética de factores pronósticos y predictivos en pacientes con LBDCG.

\section{Discusión}

En 1986, Bennett y colaboradores evaluaron el valor pronóstico del compromiso de médula ósea en 502 pacientes con diferentes histologías de linfomas no Hodgkin, diagnosticados y tratados entre 1974 y 1983. Su estudio no encontró que el compromiso de médula ósea fuese un factor pronóstico en pacientes con estadios avanzados?. En otra investigación, con esquemas de tratamiento actual, pero sin el uso de rituximab, Gronich y colaboradores demostraron un peor pronóstico en cuanto a la SG en un grupo de pacientes con linfomas no Hodgkin de bajo grado, tratados con protocolo $\mathrm{CHOP}^{9}$. Cambell y colaboradores confirmaron que el impacto negativo de este compromiso solo era evidente en casos de compromiso concordante . $^{6}$ Chung y colaboradores reportaron que el pronóstico del compromiso en médula ósea no era significativo en pacientes que usaban rituximab"11. Sin embargo, Sehn y colaboradores, en un estudio con casi 800 pacientes, encontraron que el compromiso de médula ósea sí fue

Tabla 4. Supervivencia general

\begin{tabular}{|c|c|c|c|c|c|c|}
\hline \multirow[b]{2}{*}{ Variable } & \multicolumn{3}{|c|}{ Análisis bivariado por Kaplan-Meier } & \multicolumn{3}{|c|}{ Análisis multivariado (regresión de Cox) } \\
\hline & HR & IC95\% & $\begin{array}{c}\text { Valor p } \\
\text { (Log-Rank) }\end{array}$ & HR & IC95\% & Valor $p$ \\
\hline IPI alto & 5,42 & $2,18-13,70$ & $<0,0001$ & 3,58 & $1,34-9,56$ & 0,011 \\
\hline Compromiso de médula ósea & 5,03 & $2,17-11,66$ & $<0,0001$ & 0,99 & $0,37-2,67$ & 0,994 \\
\hline Edad $>65$ años & 1,36 & $0,60-3,05$ & 0,4510 & -- & -- & -- \\
\hline Sexo masculino & 0,93 & $0,43-2,04$ & 0,8690 & -- & -- & -- \\
\hline Infección por VIH & 4,61 & $1,56-13,55$ & 0,0022 & 2,67 & $0,77-9,21$ & 0,118 \\
\hline Síntomas B & 2,08 & $0,90-4,78$ & 0,0770 & -- & -- & - \\
\hline Enfermedad bultosa & 1,58 & $0,72-3,44$ & 0,2451 & - & -- & -- \\
\hline Uso de rituximab & 0,84 & $0,35-2,01$ & 0,7060 & -- & -- & - \\
\hline Uso de radioterapia & 0,43 & $0,14-1,25$ & 0,1106 & - & - & - \\
\hline Respuesta objetiva al tratamiento & 18,02 & $8,11-40,00$ & $<0,00001$ & 16,51 & $6,57-41,45$ & $<0,0001$ \\
\hline Recaída & 7,99 & $3,47-18,40$ & $<0,00001$ & 1,74 & $0,56-5,37$ & $0,333^{*}$ \\
\hline
\end{tabular}

$p$ (modelo) $<0,00001$ prueba de asunción de riesgos proporcionales $=0,0597$. * En modelo diferente. 
un factor pronóstico independiente para supervivencia general y recaída en pacientes con linfoma B difuso de célula grande, incluso controlando el análisis multivariado por el puntaje $|\mathrm{P}|^{5}$.

A nuestro conocimiento, los resultados de este estudio son los únicos publicados con población exclusivamente latinoamericana que evalúan el valor pronóstico y predictivo de la médula ósea comprometida además del IPI. En esta población, demostramos que el compromiso de médula fue un factor predictivo fuerte de pobre respuesta al tratamiento. Nuestro análisis multivariado para supervivencia general incluyó la respuesta al tratamiento y la recaída como posibles factores asociados, características que no se han evaluado de manera regular en los modelos de Cox de los estudios previamente publicados ${ }^{3}$. En nuestro caso, como en la mayoría de las investigaciones previamente publicadas, al excluir del modelo multivariado la variable de respuesta, el compromiso de médula ósea y el puntaje de IPI fueron los únicos factores pronósticos asociados con mortalidad.

El uso de rituximab en nuestra población de manera llamativa no eliminó el riesgo incrementado que impuso el compromiso de médula ósea para la pobre respuesta al tratamiento, pero sí mejoró esta proporción. La pequeña muestra de pacientes analizados y el diseño de este estudio impiden sacar conclusiones definitivas al respecto. No obstante, a nuestro conocimiento, tampoco existen datos de estudios clínicos aleatorizados que evalúen específicamente este interrogante. En el estudio de registro del grupo GELA por Coiffier y colaboradores, 56 pacientes (28\%) tenían compromiso de médula ósea en el grupo aleatorizado a rituximab, pero no se han publicado datos sobre la respuesta y el pronóstico en este subgrupo ${ }^{12}$. Un estudio reciente ${ }^{13}$ mostró menores concentraciones valle de rituximab en pacientes con linfoma folicular con compromiso tumoral en médula ósea o enfermedad bultosa, comparado con aquellos sujetos sin estas características, lo cual está en consonancia con la pobre eficacia hallada en nuestro estudio. Otros factores, como la expresión disminuida de CD20 y la expresión aumentada de CD19, que han demostrado respuesta reducida a los esquemas con rituximab ${ }^{14}$, no se analizaron en nuestra población.

Las principales limitaciones de nuestro estudio son la recolección retrospectiva de información y el pequeño número de pacientes incluidos. A pesar de ser retrospectivo, nuestra investigación incluyó de manera cuidadosa aquellos pacientes con información adecuadamente registrada en su historia clínica, lo que garantizó una mayor confiabilidad de los datos analizados.

En nuestras instituciones, la evaluación intermedia de respuesta al tratamiento después del segundo ciclo se utiliza de manera rutinaria con tomografía computarizada y no con PET/CT. Sin embargo, los datos de muchos pacientes incluidos en este análisis no cuentan consistentemente con dicha información, por lo cual esta no se analizó. Si bien algunos datos de la literatura encuentran un valor pronóstico especialmente con el uso de $\mathrm{PET} / \mathrm{CT}^{16-20}$, todos los reportes no son consistentes en este aspecto y existen resultados divergentes, especialmente para la histología de célula B grande difusa ${ }^{21-23}$ y linfomas del manto ${ }^{24}$. Debido a que no todos los pacientes de nuestra población tenían de manera constante la realización de otros marcadores de inmunohistoquímica complementarios para subtipificar el origen centro germinal o no, ni técnicas de imagen funcionales, nuestro análisis no tuvo en cuenta estos ni otros factores pronósticos actualmente considerados potencialmente útiles, tal como el Ki67 elevado"11, alteraciones de STAT3, sobreexpresión de p5 $53^{25}$, recuento de macrófagos con anticuerpos monoclonales específicos de linaje ${ }^{26}$, expresión de CD10, $\mathrm{BCl}-6, \mathrm{BCl}-2, \mathrm{MUM1}^{27}$, y respuesta en PET al final del tratamiento ${ }^{28}$.

Nuestros datos plantean la hipótesis de que aquellos pacientes con compromiso de médula ósea, que son quienes tienen el mayor riesgo de no respuesta al tratamiento, deberían recibir esquemas diferentes que aumenten la probabilidad de respuesta, debido a la alta letalidad que implica el fallo al tratamiento, especialmente en el subgrupo de riesgo alto. Considerar un esquema con optimización de la dosis de rituximab basado en la alteración farmacocinética que impone el compromiso en médula ósea podría ser una atractiva estrategia terapéutica, que requiere confirmación en experimentos clínicos aleatorizados ${ }^{13}$. De forma alternativa, los datos recientes del estudio GELA LNH03-2B que reportan mejor SG con el uso de esquemas de quimioterapia más intensivos que el R-CHOP ${ }^{29}$ podría ser otra opción por considerar en este grupo de pacientes. Finalmente, estrategias 
como la adición de medicamentos con mecanismo de acción complementarios, como el bortezomib, resultan particularmente llamativos por las altas tasas de respuesta reportadas ${ }^{30}$.

Como conclusión, nuestro estudio confirma un valor predictivo significativo del compromiso de médula ósea para la pobre respuesta al tratamiento. La SG solo fue impactada por el puntaje IPI y la respuesta al tratamiento, evidenciando que el compromiso de médula ósea parece ser un factor predictivo de respuesta y no pronóstico independiente para SG.

\section{Referencias}

1. Globocan. Cancer incidence, mortality and prevalence worldwide in 2008. Lyon: International Agency for Research on Cancer. 2008 [cited 2011 oct $1^{\circ}$ ]. Available from: <http://globocan.iarc.

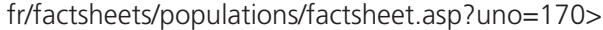

2. Ziepert $M$, Hasenclever $D$, Kuhnt $E$, Glass B, Schmitz $N$, Pfreundschuh $\mathrm{M}$, et al. Standard International prognostic index remains a valid predictor of outcome for patients with aggressive CD20+ B-cell lymphoma in the rituximab era. J Clin Oncol. 2010;28(14):2373-80.

3. A predictive model for aggressive non-Hodgkin's lymphoma. The International Non-Hodgkin's Lymphoma Prognostic Factors Project. N Engl J Med. 1993;329(14):987-94.

4. Gallamini A, Stelitano C, Calvi R, Bellei M, Mattei D, Vitolo U, et al. Peripheral T-cell lymphoma unspecified (PTCL-U): a new prognostic model from a retrospective multicentric clinical study. Blood. 2004;103(7):2474-9.

5. Sehn LH, Scott DW, Chhanabhai M, Berry B, Ruskova A, Berkahn L, et al. Impact of concordant and discordant bone marrow involvement on outcome in diffuse large B-cell lymphoma treated with R-CHOP. J Clin Oncol. 2011:29(11):1452-7.

6. Chung R, Lai R, Wei P, Lee J, Hanson J, Belch AR, et al. Concordant but not discordant bone marrow involvement in diffuse large B-cell lymphoma predicts a poor clinical outcome independent of the International Prognostic Index. Blood. 2007;110(4):1278-82.

7. Bennett JM, Cain KC, Glick JH, Johnson GJ, Ezdinli E, O'Connell MJ. The significance of bone marrow involvement in nonHodgkin's lymphoma: the Eastern Cooperative Oncology Group experience. J Clin Oncol. 1986;4(10):1462-9.

8. Hodges GF, Lenhardt TM, Cotelingam JD. Bone marrow involvement in large-cell lymphoma. Prognostic implications of discordant disease. Am J Clin Pathol. 1994;101(3):305-11.

9. Seneviratne L, Espina BM, Nathwani BN, Chan JA, Brynes RK, Levine AM. Clinical, immunologic, and pathologic correlates of bone marrow involvement in 291 patients with acquired immunodeficiency syndrome-related lymphoma. Blood. 2001;98(8):2358-63.

10. Yi SH, Xu Y, Zou DH, An G, Zhao YZ, Qi JY, et al. [Prognostic impact of bone marrow involvement (BMI) and therapies in diffuse large B cell lymphoma]. Zhonghua Xue Ye Xue Za Zhi. 2009;30(5):307-12.
11. Gaudio F, Giordano A, Perrone T, Pastore D, Curci P, Delia M, et al. High Ki67 index and bulky disease remain significant adverse prognostic factors in patients with diffuse large B cell lymphoma before and after the introduction of rituximab. Acta Haematol. 2011;126(1):44-51.

12. Coiffier B, Lepage $E$, Briere J, Herbrecht $R$, Tilly $H$, Bouabdallah $\mathrm{R}$, et al. CHOP chemotherapy plus rituximab compared with CHOP alone in elderly patients with diffuse large-B-cell lymphoma. N Engl J Med. 2002;346(4):235-42.

13. Jäger $U$, Fridrik $M$, Zeitlinger $M$, Heintel $D$, Hopfinger $G$, Burgstaller $\mathrm{S}$, et al. Rituximab serum concentrations during immunochemotherapy of follicular lymphoma correlate with patient gender, bone marrow infiltration and clinical response. Haematologica. 2012;97(9):1431-8.

14. Johnson NA, Boyle M, Bashashati $A$, Leach $S$, Brooks-Wilson A, Sehn LH, et al. Diffuse large B-cell lymphoma: reduced CD20 expression is associated with an inferior survival. Blood. 2009;113(16):3773-80.

15. Gronich N, Radnay J, Shapiro H, Manor Y, Lahav M, Lishner M. Clinical outcome of low-grade NHL patients with bone marrow involvement. Eur J Clin Invest. 2007;37(4):305-9.

16. Terasawa T, Lau J, Bardet $S$, Couturier O, Hotta T, Hutchings $\mathrm{M}$, et al. Fluorine-18-fluorodeoxyglucose positron emission tomography for interim response assessment of advanced-stage Hodgkin's lymphoma and diffuse large B-cell lymphoma: a systematic review. J Clin Oncol. 2009:27(11):1906-14.

17. Yang DH, Min JJ, Song HC, Jeong YY, Chung WK, Bae SY, et al. Prognostic significance of interim (1)(8)F-FDG PET/CT after three or four cycles of R-CHOP chemotherapy in the treatment of diffuse large B-cell lymphoma. Eur J Cancer. 2011;47(9):1312-8

18. Safar V, Dupuis J, Itti E, Jardin F, Fruchart C, Bardet S, et al. Interim [18F]fluorodeoxyglucose positron emission tomography scan in diffuse large B-cell lymphoma treated with anthracycline-based chemotherapy plus rituximab. J Clin Oncol. 2012;30(2):184-90

19. Park S, Moon SH, Park LC, Hwang DW, Ji JH, Maeng $\mathrm{CH}$, et al. The impact of baseline and interim PET/CT parameters on clinical outcome in patients with diffuse large B cell lymphoma. Am J Hematol. 2012;87(9):937-40.

20. Lanic H, Mareschal S, Mechken F, Picquenot JM, Cornic M, Maingonnat $C$, et al. Interim positron emission tomography scan associated with international prognostic index and germinal center B cell-like signature as prognostic index in diffuse large B-cell lymphoma. Leuk Lymphoma. 2012;53(1):34-42.

21. Pregno $P$, Chiappella $A$, Bello $M$, Botto $B$, Ferrero $S$, Franceschetti $S$, et al. Interim 18-FDG-PET/CT failed to predict the outcome in diffuse large B-cell lymphoma patients treated at the diagnosis with rituximab-CHOP. Blood. 2012;119(9):2066-73.

22. Cox MC, Ambrogi V, Lanni V, Cavalieri E, Pelliccia S, Scopinaro

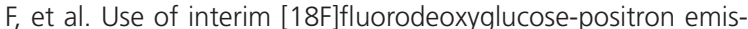
sion tomography is not justified in diffuse large B-cell lymphoma during first-line immunochemotherapy. Leuk Lymphoma. 2012;53(2):263-9.

23. Yoo C, Lee DH, Kim JE, Jo J, Yoon DH, Sohn BS, et al. Limited role of interim PET/CT in patients with diffuse large B-cell lymphoma treated with R-CHOP. Ann Hematol. 2011;90(7):797-802.

24. Mato AR, Svoboda J, Feldman T, Zielonka T, Agress $H$, Panush $D$, et al. Post-treatment (not interim) positron emission tomography-computed tomography scan status is highly predictive 
of outcome in mantle cell lymphoma patients treated with RHyperCVAD. Cancer. 2012;118(14):3565-70.

25. Cabanillas F. Non-Hodgkin's lymphoma: the old and the new. Clin Lymphoma Myeloma Leuk. 2011;11 Suppl 1:S87-90.

26. Wada N, Zaki MA, Hori Y, Hashimoto K, Tsukaguchi M, Tatsumi $Y$, et al. Tumour-associated macrophages in diffuse large Bcell lymphoma: a study of the Osaka Lymphoma Study Group. Histopathology. 2012;60(2):313-9.

27. Seki R, Ohshima K, Fujisaki T, Uike N, Kawano F, Gondo H, et al. Prognostic impact of immunohistochemical biomarkers in diffuse large B-cell lymphoma in the rituximab era. Cancer Sci. 2009;100(10):1842-7.
28. Zanoni L, Cerci JJ, Fanti S. Use of PET/CT to evaluate response to therapy in lymphoma. Q J Nucl Med Mol Imaging. 2011;55(6):633-47.

29. Récher C, Coiffier B, Haioun C, Molina TJ, Fermé C, Casasnovas $O$, et al. Intensified chemotherapy with ACVBP plus rituximab versus standard $\mathrm{CHOP}$ plus rituximab for the treatment of diffuse large B-cell lymphoma (LNH03-2B): an open-label randomised phase 3 trial. Lancet. 2011;378(9806):1858-67.

30. Ruan J, Martin P, Furman RR, Lee SM, Cheung K, Vose JM, et al. Bortezomib plus CHOP-rituximab for previously untreated diffuse large B-cell lymphoma and mantle cell lymphoma. J Clin Oncol. 2011;29(6):690-7. 